F. Bagemihl

Nagoya Math. J.

Vol. 61 (1976), 203-204

\title{
THE THREE-SEPARATED-ARC PROPERTY OF THE MODULAR FUNCTION
}

\author{
FREDERICK BAGEMIHL
}

Let $D$ be the open unit disk and $\Gamma$ be the unit circle in the complex plane, and denote the Riemann sphere by $\Omega$. If $f(z)$ is a function defined on $D$ with values belonging to $\Omega$, if $\zeta \in \Gamma$, and if $\Lambda$ is an arc at $\zeta$, then $C_{A}(f, \zeta)$ denotes the cluster set of $f$ at $\zeta$ along $\Lambda$. If there exist three mutually exclusive $\operatorname{arcs} \Lambda_{1}, \Lambda_{2}, \Lambda_{3}$ at $\zeta$ such that

$$
C_{A_{1}}(f, \zeta) \cap C_{\Lambda_{2}}(f, \zeta) \cap C_{\Lambda_{3}}(f, \zeta)=\emptyset,
$$

then $f$ is said to have the three-separated-arc property at $\zeta$.

The following theorem answers a question raised by Belna [1, p. 220] concerning the modular function $\mu(z)$ that maps $D$ onto the universal covering surface $W$ of the extended $w$-plane punctured at the points $w=0,1, \infty$.

THEOREM. The modular function $\mu(z)$ has the three-separated-arc property at every point of $\Gamma$.

Proof. For convenience and clarity, we refer the reader to the Figure, which represents the $w$-plane. The shaded lower half is the lower half-plane, the unshaded upper half is the upper half-plane. We consider three graphs, $g_{1}, g_{2}, g_{3} ; g_{1}$ is represented by the lightest lines, $g_{2}$ by the heavier lines, and $g_{3}$ by the heaviest lines.

For $j=1,2,3$, let $G_{j}$ denote the set of points on $W$ that overlie the set $g_{j}$, and let $\gamma_{j}$ be the preimage of $G_{j}$ under the mapping $\mu(z)$. One readily infers from the Figure that if $\zeta \in \Gamma$, then there are in $D$ three mutually exclusive $\operatorname{arcs} \Lambda_{1}, \Lambda_{2}, \Lambda_{3}$ at $\zeta$ such that $\Lambda_{j} \subset \gamma_{j}(j=1,2,3)$. The cluster set $C_{A_{j}}(\mu, \zeta)$ is clearly a subset of $g_{j}(j=1,2,3)$. Since it is evident that $g_{1} \cap g_{2} \cap g_{3}=\emptyset$, the theorem is proved.

Received December 2, 1975. 


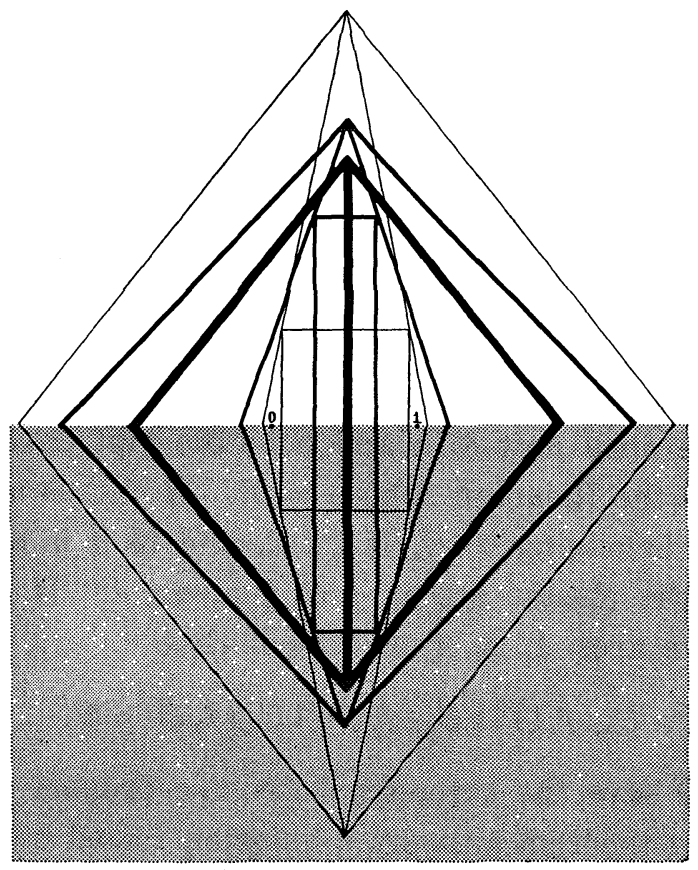

Figure

\section{REFERENCE}

[1] C. L. Belna, Intersections of arc-cluster sets for meromorphic functions, Nagoya Math. J. 40 (1970), 213-220.

University of Wisconsin-Milwaukee 